\title{
Injúria Hepática Induzida por Medicamentos em Pacientes Hospitalizados
}

\author{
Drug-induced Liver Injury in Hospitalized Patients \\ Lesión Hepática Inducida por Fármacos en Pacientes Hospitalizados
}

Recebido: 12/11/2021 | Revisado: 20/11/2021 | Aceito: 25/11/2021 | Publicado: 07/12/2021

\author{
Simone Tomás Gonçalves \\ ORCID: https://orcid.org/0000-0003-3565-631X \\ Universidade Estadual de Maringá, Brasil \\ E-mail: stgoncalves@uem.br \\ Estela Louro \\ ORCID: https://orcid.org/0000-0002- 0759-4126 \\ Universidade Estadual de Maringá, Brasil \\ E-mail: elouro@uem.br \\ Raíssa Bocchi Pedroso \\ ORCID: https://orcid.org/0000-0002-0076-1032 \\ Universidade Estadual de Maringá, Brasil \\ E-mail: raissap@gmail.com \\ Ana Luiza Pelissari Pessanha de Paula Soares \\ ORCID: https://orcid.org/0000-0002-6501-3799 \\ Santa Casa de Maringá, Brasil \\ E-mail: aninhappps@gmail.com \\ Roberto Kenji Nakamura Cuman \\ ORCID: https://orcid.org/0000-0002-4906-887X \\ Universidade Estadual de Maringá, Brasil \\ E-mail: rkncuman@uem.br
}

\section{Resumo}

Objetivo: Realizar uma revisão da literatura para identificar quais os medicamentos que mais causam injúria hepática induzida por medicamentos (DILI - que vem do inglês Drug Induced Liver Injury) em pacientes hospitlalizados. Metodologia: Foi realizada uma revisão da literatura por meio de busca sistemática de artigos sobre DILI em pacientes hospitalizados. Esta pesquisa seguiu a recomendação PRISMA-P (Principais Itens para Relatar Revisões Sistemáticas e Meta- Análises) e foram pesquisadas as seguintes bases de dados eletrônicas: PubMed, Web of Science, Scopus e Lilacs. Resultados: Inicialmente foi identificado um total de 454 artigos e após revisão do texto completo, foram selecionados 27 artigos. Todos os artigos selecionados consideraram o valor da enzima hepática alanina aminotransferase elevado para a caracterização da DILI. Foi identificado relatos de uma grande variedade de medicamentos, como prováveis causas de DILI, em pacientes hospitalizados. Conclusão: Os relatos de medicamentos associados a DILI, de acordo com a classificação ATC, foram: 85\% (23) de fármacos do grupo J, 37\% (10) do grupo $\mathrm{M}, 33,3 \%$ (9) do grupo $\mathrm{N}$, e 33,3\% (9) do grupo C. O grupo J, que abrange fármacos anti-infecciosos, foi o que teve maior número de relatos, sendo 47,8\% (11) de antibióticos, 47,8\% (11) de antituberculostáticos e 34,7 $\%$ (8) de antifúngicos. Destes, destacaram -se os Beta-lactâmicos, em especial a Amoxacilina-Clavulanto, Rifampicina, Isoniazida, Pirazinamida e Fluconazol.

Palavras-chave: Injúria hepática induzida por medicamentos; Reações adversas associadas a medicamentos; Pacientes hospitalizados; Hospital.

\begin{abstract}
Objective: To conduct a literature review to identify which drugs cause the most drug induced liver injury (DILI) in hospitalized patients. Methodology: A literature review was performed through a systematic search for articles on DILI in hospitalized patients. This research followed the PRISMA-P (Main Items for Reporting Systematic Reviews and Meta-Analyses) recommendation and the following electronic databases were searched: PubMed (National Library of Medicine, National Institutes of Health), Web of Science, Scopus and Lilacs (Latin American and Caribbean Literature in Health Sciences). Results: Initially, a total of 454 articles were identified and after reviewing the full text, 27 articles were selected. All articles considered the value of the liver enzyme alanine aminotransferase increased for DILI characterization. Reports of a wide variety of medications, as probable causes of DILI, were identified in hospitalized patients. Conclusion: Among the reports of drugs found associated with DILI, those with the most frequent occurrence, according to the ATC classification, were: 85\% (23) of drugs in J group, 37\% (10) in M group, 33.3\% (9) in $\mathrm{N}$ group and 33.3\% (9) in $\mathrm{C}$ group. The $\mathbf{J}$ group, which covers anti-infective drugs, had the highest number of reports, with $47.8 \%$ (11) of antibiotics, $47.8 \%$ (11) of antituberculostatics and 34.7\% (8) of antifungals. Beta-lactams group, especially Amoxacillin-Clavulanate, Rifampicin, Isoniazid, Pyrazinamide and Fluconazole were stood out.
\end{abstract}


Keywords: Chemical and drug induced liver injury; Drug-related side effects and adverse reactions; Patients; Hospitals.

\begin{abstract}
Resumen
Objetivo: Realizar una revisión de la literatura para identificar los fármacos que más causan daño hepático inducido por fármacos (DILI - que proviene del inglés Drug Induced Liver Injury) en pacientes hospitalizados. Metodología: Se realizó una revisión de la literatura mediante una búsqueda sistemática de artículos sobre DILI en pacientes hospitalizados. Esta investigación siguió la recomendación PRISMA-P (Elementos principales para informar revisiones sistemáticas y metaanálisis) y se realizaron búsquedas en las siguientes bases de datos electrónicas: PubMed, Web of Science, Scopus y Lilacs. Resultados: Inicialmente se identificaron un total de 454 artículos y luego de revisar el texto completo se seleccionaron 27 artículos. Todos los artículos seleccionados consideraron alto el valor de la enzima hepática alanina aminotransferasa para la caracterización de DILI. Se han identificado informes de una amplia variedad de medicamentos como posibles causas de DILI en pacientes hospitalizados. Conclusión: Los reportes de fármacos asociados a DILI, según la clasificación ATC, fueron: 85\% (23) de fármacos del grupo J, 37\% (10) del grupo $\mathrm{M}(10), 33,3 \%$ (9) del grupo $\mathrm{N}$ y 33,3\% (9) del grupo C. El grupo J, que incluye fármacos antiinfecciosos, tuvo el mayor número de notificaciones, con 47,8\% (11) de antibióticos, 47,8\% (11) de antituberculosos y 34,7\% (8) de antifúngicos. De estos, se destacaron los Betalactámicos, especialmente AmoxicilinaClavulanto, Rifampicina, Isoniazida, Pirazinamida y Fluconazol.

Palabras clave: Enfermedad hepática inducida por sustancias y drogas; Efectos colaterales y reacciones adversas relacionados con medicamentos; Pacientes; Hospitales.
\end{abstract}

\title{
1. Introdução
}

A lesão hepática induzida por medicamentos (DILI - que vem do inglês drug induced liver injury) é uma reação adversa incomum, com sua frequência variando de 1 em cada 10.000 a 1 em cada 100.000 casos de efeitos adversos à medicamentos, mas potencialmente letal (Larrey, 2002; Björnsson, 2010). Embora a maioria dos episódios de DILI sejam autolimitados, com resolução após a retirada do agente suspeito, é a causa mais comum de insuficiência hepática aguda nos EUA, Europa e em vários países (Ostapowicz et al., 2002; Lee, 2013; Fontana et al., 2014; Kullak-Ublick et al., 2017, Bessone et al., 2019). A DILI é responsável por 11\% -14\% dos casos de falência hepática aguda, geralmente atribuídos ao uso de antiinfecciosos, suplementos fitoterápicos e dietéticos, agentes cardiovasculares e do sistema nervoso central, nos EUA e na China (Chalasani et al., 2015; Ganger et al., 2018; Shen et al., 2019). Na França e na Islândia, a DILI ocorre com uma incidência anual de cerca de 14-19 por 100.000 habitantes (Björnsson et al., 2013; Sgro et al., 2002). Em um estudo realizado no Brasil, a maioria dos casos de insuficiência hepática aguda estavam ligados a etiologia indeterminada, DILI e hepatite autoimune. DILI foi a causa mais comum de falência hepática aguda em alguns estados do Brasil particularmente as regiões Sul e Sudeste (Santos et al., 2021).

O diagnóstico de DILI ainda é uma questão desafiadora na prática clínica e depende da exclusão de outras causas. As enzimas hepáticas anormais detectadas nos exames laboratoriais, na ausência de doenças hepáticas mais comuns, devem sempre levar o clínico a suspeitar de DILI. O algoritmo CIOMS/RUCAM (Council for International Organizations of Medical Science/Roussel Uclaf Causality Assessment Method) específico para o fígado, embora imperfeito, ainda é o melhor para avaliação de causalidade da DILI (García-Cortés et al., 2011; Danan \& Teschke, 2016, Danan \& Teschke, 2019).

Ao avaliar um paciente com doença hepática de início recente, os medicamentos, incluindo aqueles prescritos, automedicação, suplementos dietéticos, vitaminas e medicamentos fitoterápicos, devem sempre ser considerados como uma causa possível de DILI. Assim, a identificação de pacientes em risco para uma potencial reação adversa pode ser benéfica, pois a intervenção precoce pode melhorar os desfechos dos tratamentos dos pacientes (Chitturi \& Farrell, 2011; Danan \& Teschke, 2016).

A DILI também adiciona um risco significativo ao desenvolvimento de medicamentos, pois só é detectada no final dos ensaios clínicos de fase III ou mesmo depois de um medicamento ter sido comercializado (fase IV); além de que, a gravidade da hepatotoxicidade levou as agências reguladoras a retirar vários medicamentos do mercado (Goldkind \& Laine, 
2006; Jee et al., 2021).

Considerando que estudos de ocorrência de DILI em pacientes hospitalizados são escassos, o objetivo deste trabalho foi identificar quais os medicamentos que mais causam DILI em pacientes hospitalizados.

\section{Metodologia}

Foi realizada uma revisão da literatura por meio de busca sistemática de artigos, com a finalidade de sintetizar os principais resultados dos estudos sobre medicamentos associados à DILI em pacientes hospitalizados, cuja análise foi de caráter exploratório (Mendes, et al., 2008; Sousa et al, 2017; Pereira et al., 2018). A revisão foi composta por seis etapas: elaboração da pergunta norteadora; busca na literatura; coleta de dados; análise crítica dos estudos incluídos; discussão dos resultados; apresentação da revisão (Souza et al., 2010).

Esta pesquisa é relatada de acordo com a recomendação PRISMA-P (Principais Itens para Relatar Revisões Sistemáticas e Meta- Análises) (Moher et al., 2019). A estratégia PICOS, que representa um acrônimo para Paciente, Intervenção, Comparação, Resultados e Tipos de Estudos, foi empregada para a construção da pergunta de pesquisa (Santos et al., 2007). Assim, foi definida a pergunta norteadora, utilizando a estratégia PICOS: Quais os medicamentos que mais causam DILI em pacientes hospitalizados? Considerando PICOS, P representa pacientes hospitalizados; I, administração de medicação por alguma via; C, reação adversa; O, Dano hepático: S, estudos encontrados nas bases de dados eletrônicas. Além disso, referências adicionais dos artigos originais foram levantadas a fim de identificar outras publicações de interesse. Como há poucas publicações sobre DILI em pacientes hospitalizados, foram incluídos todos os artigos encontrados, considerando os critérios de inclusão.

Os critérios de inclusão considerados foram estudos em humanos de pacientes hospitalizados que apresentaram lesão hepática como reação adversa devido à administração de medicamentos, escritos nos idiomas português, inglês, espanhol e francês. Foram excluídos estudos in vitro e in vivo, artigos que utilizaram fitoterapia, estudos de revisão, revisão sistemática, meta-análise, patente, estudo comparativo, comentários, editorial, congresso, revisão integrativa-abrangente e nenhum resumo disponível.

A pesquisa foi realizada utilizando-se as seguintes bases de dados eletrônicas: PubMed, Web of Science, Scopus e Lilacs. Para fornecer uma busca mais completa na literatura, as listas de referências dos artigos originais que foram incluídos na revisão foram selecionadas manualmente. Esta etapa do estudo foi altamente relevante, porque permitiu a identificação de publicações que não foram encontradas nas buscas dos bancos de dados de acordo com os descritores e estratégias de busca predefinidas.

Nas buscas pelos artigos, usou-se os termos MesSH (Medical Subject Headings) e os Descritores em Ciências da Saúde (DeCS):

PubMed: "Drug-Related Side Effects and Adverse Reactions"[Mesh] OR "Medication Errors"[Mesh] AND "Chemical and Drug Induced Liver Injury"[Mesh] AND "Patients"[Mesh] OR "Hospitals"[Mesh]) OR "Hospitalization"[Mesh]. Com filtros: inglês, português, francês e espanhol; estudo em humanos; e com resumo.

Web of Science: \#1 TS=Drug-Related Side Effects AND TS=Adverse Reactions; \#2 TS=Medication Errors; \#3 TS=Chemical AND TS=Drug Induced Liver Injury; \#4 TS= Hospitalization; \#5 TS= Patients; \#6 TS= liver injury; Grupo1: \#6 OR \#5 OR \#4; Grupo 2: \#2 OR \#1; Grupo 3: \#6 OR \#3 = 65.394; Grupo 1 AND Grupo 2 AND Grupo 3.

Scopus: ("Drug-Related Side Effects and Adverse Reactions") OR ("Medication Errors") AND ("Chemical and DrugInduced Liver Injury") AND ("Patients") OR ("Hospitals") OR ("Hospitalization") AND (LIMIT-TO (LANGUAGE "English") OR LIMIT-TO (LANGUAGE, "French") OR LIMIT-TO (LANGUAGE, "Spanish").

Lilacs: Doença Hepática Induzida por Substâncias e Drogas [Descritor de assunto] OR Efeitos Colaterais e Reações 
Adversas Relacionados a Medicamentos [Descritor de assunto] OR Hospitalizações [Descritor de assunto].

A busca na literatura em cada base de dados foi realizada em 26 de março de 2021, sendo encontrado um total de 454 artigos. Para o total de artigos selecionados foram aplicadas três etapas de identificação e seleção: identificação dos artigos nas bases de dados; seleção dos artigos por meio da leitura dos títulos e resumos; e elegibilidade dos artigos de acordo com a pergunta de pesquisa.

Para a seleção dos artigos, quatro pesquisadores independentes avaliaram os estudos previamente identificados. Nos casos em que um consenso não foi obtido, um quinto examinador foi consultado e, com base no seu parecer, decidiu-se pela inclusão ou não do artigo. A seleção final das publicações foi realizada de comum acordo entre os pesquisadores. Os pesquisadores realizaram a extração de dados de forma independente e as discordâncias foram resolvidas por especialistas. Coletou-se características gerais dos estudos, como ano de publicação, autores, cidade e país, características do paciente (idade, sexo), nome do medicamento administrado ou a classe terapêutica, bem como a reação adversa e principais conclusões. Todos os títulos e resumos inicialmente selecionados foram exportados de cada base de dados para o programa Endnote versão 9 (Thomson, Reuters, Carlsbad, Usa), para realizar a análise.

Com os resultados sintetizados e agrupados, procedeu-se com a análise criteriosa, detalhada e descritiva, confrontando os dados com o conhecimento teórico, em busca de integralização dos resultados (Whittemore \& Knafl, 2005). Por fim, realizou-se uma análise crítica dos estudos de modo a conhecer o enfoque da produção científica sobre as injúrias hepáticas induzidas por medicamentos em pacientes hospitalizados.

A interpretação dos dados ocorreu de forma crítica e imparcial, a fim de permitir apresentações de possíveis explicações para os resultados encontrados, fossem eles convergentes ou conflitantes, com base na literatura disponível.

\section{Resultados e Discussão}

Inicialmente foi identificado um total de 454 artigos de estudos em pacientes hospitalizados que tiveram DILI, dos quais 8 artigos foram excluídos, pois 6 eram duplicados e 1 triplicado. Com base no título e na análise do resumo, 351 artigos foram excluídos, sendo selecionados 95 para à leitura na íntegra. Após análise criteriosa, 21 foram elegíveis e da revisão destas referências, acrescentou-se 6, totalizando 27 artigos (Figura 1). 
Research, Society and Development, v. 10, n. 16, e70101623236, 2021

(CC BY 4.0) | ISSN 2525-3409 | DOI: http://dx.doi.org/10.33448/rsd-v10i16.23236

Figura 1. Fluxograma do processo de identificação e seleção dos artigos incluídos na revisão.

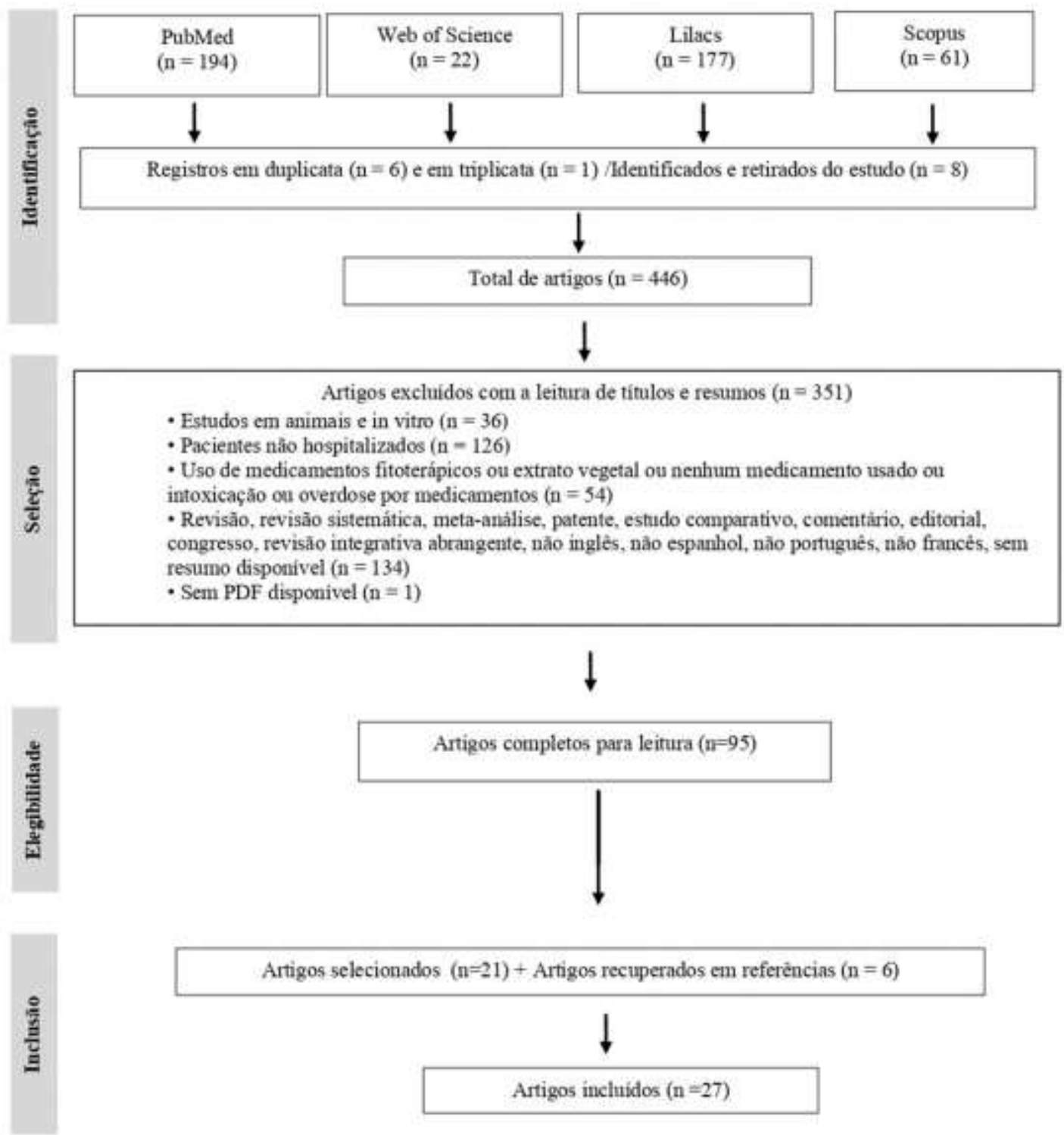

Fonte: Gonçalves, et al. (2021).

Os principais achados dos artigos selecionados são apresentados na Tabela 1. O período de publicação dos estudos ocorreu de 1994 a 2020. 
Tabela 1: Características dos estudos observacionais de DILI.

\begin{tabular}{|c|c|c|c|c|c|}
\hline Artigo & $\begin{array}{l}\text { Tipo de } \\
\text { Estudo }\end{array}$ & $\begin{array}{l}\text { Cidade/ } \\
\text { País }\end{array}$ & $\begin{array}{l}\text { Período do } \\
\text { Estudo }\end{array}$ & Comorbidades & Resultados \\
\hline $\begin{array}{l}\text { GEARHART, } \\
\text { M. O., } 1994\end{array}$ & $\begin{array}{l}\text { Relato de } \\
\text { caso }\end{array}$ & Sem dados & Sem dados & $\begin{array}{l}\text { Anemia, Trombocitopenia, Coagulopatia, } \\
\text { Endocardite, Insuficiência cardíaca congestiva. }\end{array}$ & $\begin{array}{l}\text { O paciente morreu de insuficiência } \\
\text { cardíaca. }\end{array}$ \\
\hline $\begin{array}{l}\text { OZICK, L. A. } \\
\text { et al., } 1995\end{array}$ & Prospectivo & $\begin{array}{l}\text { New York, } \\
\text { USA }\end{array}$ & $\begin{array}{l}\text { Maio à Nov. } \\
1990\end{array}$ & Tuberculose, HIV. & Descontinuação do medicamento. \\
\hline $\begin{array}{l}\text { SCHABERG, } \\
\text { T., } 1996\end{array}$ & Retrospectivo & Sem dados & 1990 a 1994 & $\begin{array}{l}\text { Tuberculose, Lesões hepáticas, Hepatites, } \\
\text { Diabetes mellitus, HIV. }\end{array}$ & Descontinuação do medicamento. \\
\hline $\begin{array}{l}\text { BAGHERI, H. } \\
\text { et al., 2000. }\end{array}$ & Prospectivo & $\begin{array}{l}\text { Toulouse, } \\
\text { França }\end{array}$ & $\begin{array}{l}\text { Junho à } \\
\text { Out. } 1997\end{array}$ & $\begin{array}{l}\text { Doenças cardíacas, Hepatite, } \\
\text { obstrutiva, Doenças neoplásicas, Hepatite } \\
\text { autoimune, Doença de Paget, Doença de } \\
\text { Crohn, Amilose hepática, Choque } \\
\text { hipovolêmico, Hepatite de nódulo } \\
\text { regenerativo, Diabetes insulinodependente. }\end{array}$ & $\begin{array}{l}\text { Prolongamento da hospitalização; } \\
\text { Risco de vida (1 paciente). }\end{array}$ \\
\hline $\begin{array}{l}\text { FISCHER, M. } \\
\text { A. et al., } 2005\end{array}$ & $\begin{array}{c}\text { Coorte } \\
\text { Retrospectivo }\end{array}$ & $\begin{array}{l}\text { Boston, } \\
\text { Massachusett } \\
\text { s, USA }\end{array}$ & $\begin{array}{l}\text { Jan. } 2000 \text { à } \\
\text { Dec. } 2001\end{array}$ & $\begin{array}{l}\text { Leucemia aguda, Leucemia crônica, Linfoma, } \\
\text { Mieloma. }\end{array}$ & Piora das condições do paciente \\
\hline $\begin{array}{l}\text { MEIER, Y. et } \\
\text { al., } 2005\end{array}$ & $\begin{array}{c}\text { Coorte } \\
\text { Retrospectivo }\end{array}$ & Suíça & $\begin{array}{l}\text { Jan. } 1996 \text { à } \\
\text { Dec. } 2000\end{array}$ & $\begin{array}{l}\text { Cirrose, Tumores hepáticos, Hepatite viral } \\
\text { hepatotrópica aguda, Hepatite B ou C crônica, } \\
\text { Insuficiência cardíaca congestiva, Hipotensão } \\
\text { grave transitória, Infecções agudas, Colestase } \\
\text { extra-hepática, Doença autoimune. }\end{array}$ & $\begin{array}{l}\text { Recuperado, após a suspensão do } \\
\text { medicamento. }\end{array}$ \\
\hline $\begin{array}{l}\text { MONTERO, } \\
\text { A. R. et al., } \\
\quad 2005\end{array}$ & Prospectivo & $\begin{array}{l}\text { Seville, } \\
\text { Espanha }\end{array}$ & $\begin{array}{l}\text { Junho } 2002 \\
\text { à Julho } \\
2003\end{array}$ & Sem dados & DILI: 6 graves e 29 leves \\
\hline $\begin{array}{l}\text { COCA, N. S. } \\
\text { M. at al, } 2010\end{array}$ & Caso-controle & $\begin{array}{l}\text { Belo } \\
\text { Horizonte, } \\
\text { Brasil }\end{array}$ & 2005 à 2007 & $\begin{array}{l}\text { Tuberculose, HIV, Diabetes mellitus, } \\
\text { Hipertensão arterial sistêmica, Insuficiência } \\
\text { cardíaca, Insuficiência renal crônica. }\end{array}$ & Descontinuação do medicamento. \\
\hline $\begin{array}{c}\text { LIMA \& } \\
\text { MELO, 2012 }\end{array}$ & Caso-controle & Recife, Brasil & $\begin{array}{l}\text { Jan. } 2004 \text { à } \\
\text { Out. } 2007\end{array}$ & Tuberculose, HIV. & $\begin{array}{l}\begin{array}{l}\text { Manutenção do tratamento, } \\
\text { interrupção } \\
\text { descontinuação do medicamento }\end{array} \\
\text { temporária, }\end{array}$ \\
\hline $\begin{array}{l}\text { SIDERI, G. et } \\
\text { al., } 2012\end{array}$ & Prospectivo & $\begin{array}{l}\text { Athens, } \\
\text { Grécia }\end{array}$ & 2005 a 2008 & $\begin{array}{l}\text { Malignidade, Leucemia linfoblástica aguda, } \\
\text { Tumor encefálico, Leucemia mieloide aguda e } \\
\text { Linfoma. }\end{array}$ & $\begin{array}{l}\text { Descontinuação do medicamento ( } 2 \\
\text { casos) }\end{array}$ \\
\hline $\begin{array}{l}\text { XU, H.-M. et } \\
\text { al., } 2012\end{array}$ & Retrospectivo & $\begin{array}{l}\text { Zhejiang, } \\
\text { China }\end{array}$ & $\begin{array}{l}\text { Jan. } 2010 \text { à } \\
\text { Dec. } 2010\end{array}$ & Lesão hemodinâmica, Malignidade & $\begin{array}{l}\text { Recuperado: } 10(40 \%) \text {; Melhora } \\
\text { antes da alta: } 15(60 \%) \text {. }\end{array}$ \\
\hline $\begin{array}{l}\text { LEE, T. et al., } \\
2013\end{array}$ & Retrospectivo & Seoul, Coreia & $\begin{array}{l}\text { Jan. } 2008 \text { à } \\
\text { Feb. } 2011 .\end{array}$ & $\begin{array}{l}\text { DRESS, Stevens-Johnson, Síndrome, } \\
\text { Necrólise epidérmica tóxica, Erupção por } \\
\text { droga fixa, Púrpura, Pustulose exantemática } \\
\text { generalizada aguda, Eritrodermia, Erupção } \\
\text { bolhosa por droga. }\end{array}$ & $\begin{array}{l}\text { Aumento da mortalidade causada } \\
\text { por hipersensibilidade tardia ao } \\
\text { medicamento em pacientes com } \\
\text { disfunção hepática. }\end{array}$ \\
\hline $\begin{array}{l}\text { BOHM, N. et } \\
\text { al., } 2014\end{array}$ & $\begin{array}{l}\text { Relato de } \\
\text { caso/ Coorte } \\
\text { Retrospectiva }\end{array}$ & $\begin{array}{l}\text { South } \\
\text { Carolina, } \\
\text { USA }\end{array}$ & $\begin{array}{c}\text { Coorte: } \\
\text { Maio 2008 à } \\
\text { Março } 2013\end{array}$ & $\begin{array}{l}\text { Coorte: Síndrome do enxerto, Infecção fúngica } \\
\text { hepática, Cirrose biliar primária, Choque } \\
\text { hepático relacionado com hipotensão. }\end{array}$ & $\begin{array}{l}\text { Relato de caso: recuperado após a } \\
\text { suspensão do medicamento. } \\
\text { Coorte: não sério }\end{array}$ \\
\hline $\begin{array}{l}\text { CHALCO- } \\
\text { AGUATE, M. } \\
\text { et al., } 2014\end{array}$ & $\begin{array}{c}\text { Coorte } \\
\text { Retrospectiva }\end{array}$ & Peru & 2010 & Sem dados & $\begin{array}{l}\text { Alta frequência de DILI em } \\
\text { pacientes com leishmaniose } \\
\text { cutânea com } \\
\text { estibogluconato de sódio IV. }\end{array}$ \\
\hline $\begin{array}{l}\text { DOUROS, A. } \\
\text { et al., } 2015\end{array}$ & Caso-controle & $\begin{array}{l}\text { Berlin, } \\
\text { Alemanha }\end{array}$ & $\begin{array}{l}\text { Out. } 2002 \text { à } \\
\text { Dec. } 2011\end{array}$ & Sem dados & $\begin{array}{l}\text { Mortalidade: } 1(1,3 \%) \text {. } \\
\text { Lesão hepática como causa certa de } \\
\text { morte }\end{array}$ \\
\hline $\begin{array}{l}\text { WOO, H. J. et } \\
\text { al, } 2015\end{array}$ & Retrospectivo & Seoul, Coreia & $\begin{array}{l}\text { Jan. } 2012 \text { à } \\
\text { Julho } 2014\end{array}$ & $\begin{array}{l}\text { Hipertensão, Diabetes, Dislipidemia, Enfarte } \\
\text { cerebral, } \\
\text { Hemorragia cerebral, Fibrilação atrial. }\end{array}$ & Sem dados \\
\hline $\begin{array}{l}\text { SCHNEEWEI } \\
\text { SS, S. et al., } \\
2016\end{array}$ & Coorte & USA & $\begin{array}{l}\text { Jan. } 2005 \text { à } \\
\text { Dec. } 2012 .\end{array}$ & $\begin{array}{l}\text { Câncer, Doenças hematopoiéticas, HIV } \\
\text { positivo, Imunodeficiência congênita, Doença } \\
\text { renal crônica, Doença hepática crônica, } \\
\text { Doença cardiovascular, Diabetes, Hipertensão, } \\
\text { Doença respiratória crônica. }\end{array}$ & DILI pela Micafungina. \\
\hline $\begin{array}{l}\text { FRIEDRICH, } \\
\text { M.-E. et al., } \\
2016\end{array}$ & Retrospectivo & $\begin{array}{l}\text { Áustria, } \\
\text { Alemanha e } \\
\text { Suíça }\end{array}$ & 1993 a 2011 & $\begin{array}{l}\text { Esquizofrenia, Depressão, Lesões hepáticas } \\
\text { pré-existentes. }\end{array}$ & $\begin{array}{l}\text { Dosagem recuperada, reduzida, } \\
\text { retirada do medicamento, } \\
\text { descontinuação do medicamento }\end{array}$ \\
\hline $\begin{array}{l}\text { ISA, S. E. et } \\
\text { al., } 2016\end{array}$ & $\begin{array}{c}\text { Coorte } \\
\text { Retrospectiva }\end{array}$ & Jos, Nigeria & $\begin{array}{l}\text { Jan/2013 à } \\
\text { Junho/2013 }\end{array}$ & Tuberculose pulmonar ou extrapulmonar, HIV. & $\begin{array}{l}\text { DILI devido a anti-TBs de primeira } \\
\text { linha }\end{array}$ \\
\hline $\begin{array}{l}\text { SALOOJEE, } \\
\text { A. et al., } 2017\end{array}$ & Retrospectivo & $\begin{array}{l}\text { Kwazulu- } \\
\text { Natal, África } \\
\text { do Sul }\end{array}$ & $\begin{array}{l}\text { Jan. à Dec. } \\
2012\end{array}$ & $\begin{array}{l}\text { Trauma contuso, Trauma penetrante, Trauma } \\
\text { hepático. }\end{array}$ & $\begin{array}{l}\text { DILI em pacientes com trauma, } \\
\text { leva ao aumento da mortalidade, } \\
\text { aumento do risco de disfunção de } \\
\text { outros órgãos e tempo de } \\
\text { internação prolongado. }\end{array}$ \\
\hline $\begin{array}{l}\text { VEKEMAN, } \\
\text { F., } 2018\end{array}$ & $\begin{array}{c}\text { Coorte } \\
\text { Retrospectiva }\end{array}$ & USA & 2006 a 2013 & $\begin{array}{l}\text { Varizes esofágicas, Doença hepática, Diabetes, } \\
\text { Endocardite, Refluxo gastroesofágico, } \\
\text { Hipertensão, Falências de órgãos, Sepse ou } \\
\text { choque séptico, Disfunção renal. }\end{array}$ & $\begin{array}{l}\text { Anidulafungina: Recuperado } 198 \\
(31,3 \%) \text {; Não recuperado } 423 \\
(66,8 \%) \text {; Desconhecido: } 12(1,9 \%) \text {. } \\
\text { Caspofungina: } 316 \text { recuperada }\end{array}$ \\
\hline
\end{tabular}




\begin{tabular}{|c|c|c|c|c|c|}
\hline & & & & & 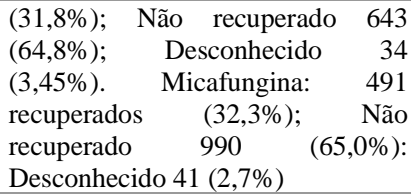 \\
\hline $\begin{array}{l}\text { EL KAHI, C. } \\
\text { et al., } 2018\end{array}$ & $\begin{array}{l}\text { Relato de } \\
\text { caso }\end{array}$ & Sem dados & Sem dados & Gota & $\begin{array}{l}\text { Recuperado, Descontinuação do } \\
\text { medicamento }\end{array}$ \\
\hline $\begin{array}{l}\text { FANG, W. C. } \\
\text { et al., } 2018\end{array}$ & $\begin{array}{c}\text { Coorte } \\
\text { Retrospectiva }\end{array}$ & $\begin{array}{l}\text { Melbourne, } \\
\text { Victoria, } \\
\text { Australia }\end{array}$ & $\begin{array}{l}\text { Jan. } 2004 \text { à } \\
\text { Agosto } \\
2014\end{array}$ & $\begin{array}{l}\text { Síndrome de Stevens-Johnson, } \\
\text { epidérmica tóxica, DRESS, } \\
\text { exantise } \\
\text { exantemática generalizada aguda, Dermatose } \\
\text { bolhosa por IgA linear, Eritema multiforme. }\end{array}$ & Prolongamento da hospitalização \\
\hline $\begin{array}{l}\text { WANG, S. et } \\
\text { al., } 2018\end{array}$ & Retrospectivo & $\begin{array}{l}\text { Zhejiang, } \\
\text { China }\end{array}$ & 2010 a 2016 & $\begin{array}{l}\text { Tuberculose pulmonar ou extrapulmonar, HIV, } \\
\text { Hepatite viral crônica, Doença hepática } \\
\text { gordurosa, Esquistossomose hepática, Diabetes } \\
\text { mellitus. }\end{array}$ & $\begin{array}{l}\text { Descontinuação do medicamento, } \\
\text { Falência hepática aguda, } \\
\text { Mortalidade: } 9,68 \% \text {. }\end{array}$ \\
\hline $\begin{array}{l}\text { CANO- } \\
\text { PANIAGUA, } \\
\text { A. et al., 2019 }\end{array}$ & Prospectivo & $\begin{array}{l}\text { Medellín, } \\
\text { Colombia }\end{array}$ & $\begin{array}{l}\text { Nov. } 2015 \text { à } \\
\text { Nov. } 2016\end{array}$ & $\begin{array}{l}\text { Artrite reumatoide, Insuficiência cardíaca, } \\
\text { Histoplasmose, Abscesso hepático, Infecções } \\
\text { virais. }\end{array}$ & Descontinuação do medicamento \\
\hline $\begin{array}{l}\text { WORLAND, } \\
\text { T. et al., 2020 }\end{array}$ & $\begin{array}{l}\text { Retrospectivo } \\
\text { Caso-controle }\end{array}$ & $\begin{array}{l}\text { Melbourne, } \\
\text { Australia }\end{array}$ & $\begin{array}{l}\text { Nov. } 2011 \text { à } \\
\text { Junho } 2017\end{array}$ & $\begin{array}{l}\text { Diabetes mellitus, Doença autoimune, } \\
\text { Hiperlipidemia, Doença hepática. }\end{array}$ & Prolongamento da hospitalização \\
\hline $\begin{array}{l}\text { ALI, N. et al, } \\
2020\end{array}$ & Coorte & India & $\begin{array}{l}\text { Set. } 2017 \text { à } \\
\text { Agosto } \\
2019\end{array}$ & $\begin{array}{l}\text { Tuberculose, Doença hepática pré-existente, } \\
\text { HIV }\end{array}$ & Descontinuação do medicamento \\
\hline
\end{tabular}

Fonte: Gonçalves, et al. (2021).

Nove estudos foram conduzidos no Continente Americano: 5 nos EUA (Ozick et al., 1995, Fischer et al., 2005; Bohm, et al., 2014; Schneeweiss et al., 2016, Vekeman et al., 2018); 1 no Peru (Chalco-Aguate et al., 2014); 1 na Colombia (Cano-Paniagua et al., 2019) e 2 no Brasil (Coca et al., 2010, Lima \& Melo, 2012). Sete estudos foram realizados na Europa: 1 na França (Bagheri et al., 2000), 1 na Suíça (Meier et al., 2005), 1 na Espanha (Montero et al., 2005), 1 na Grécia (Sideri et al., 2012), 2 na Alemanha (Douros et al., 2014; Friedrich et al., 2016) e 1 na Áustria (Friedrich. et al., 2016). Cinco foram realizados na Ásia: 2 na China (Xu et al., 2012; Wang et al., 2018), 2 na Coreia do Sul (Lee et al., 2013; Woo et al., 2015) e 1 na Índia (Ali et al., 2020). Dos estudos restantes, 2 ocorreram na África (Isa, et al., 2016; Saloojee et al., 2017), 2 na Austrália (Fang et al., 2018; Worland, et al., 2020) e em 2 não foi identificado o local (Gearhat, 1994; Schaberg et al.).

Quanto ao delineamento da pesquisa, evidenciou-se somente estudos observacionais, sendo 33,3\% coorte retrospectivo, $26 \%$ retrospectivo, 18,5\% prospectivo, $14,8 \%$ caso controle e 7, $4 \%$ relato de caso. Propôs-se, com esta revisão, investigar a produção científica que identifica quais medicamentos estão causando DILI em pacientes hospitalizados. Destaca-se que, a maioria dos artigos selecionados apresentam estudos retrospectivos de ocorrência do evento adverso em pacientes hospitalizados, na base de dados, o que pode limitar a associação de fatores relacionados às lesões geradas pelos eventos neste período.

Dos 27 artigos seleccionados, a maioria (24) relataram comorbidades, descritas na Tabela1; e em apenas dois (Douros et al., 2014; Wang, et al., 2018) houve relato de mortalidade tendo como causa a DILI.

Quanto aos critérios utilizados para a caracterização da DILI (Tabela 2), observamos que não houve concenso, apesar de que todos os artigos consideraram o valor da enzima hepática alanina aminotransferase (ALT que vem do inglês alanine aminotransferase) elevado. Sete artigos (Meier et al., 2005; Xu et al., 2012; Woo et al., 2015; Fang et al., 2018; Wang et al., 2018; Cano-Paniagua et al., 2019; Worland et al., 2020) utilizaram o algorítmo de causalidade de RUCAM /CIOMS e 4 usaram algoritmo de Narranjo (Bohm et al., 2014; El Kahi et al., 2018; Fang et al., 2018; Worland et al., 2020), que não é específico para lesão hepática e pode ser usado para qualquer tipo de reação adversa a medicamento. Um artigo (Montero et al., 2005) utilizou o Sistema Maria e Victorino, que consiste numa modificação do RUCAM. 
Tabela 2: Critérios de inclusão de DILI.

\begin{tabular}{|c|c|c|}
\hline ARTIGO & $\begin{array}{l}\text { TESTES } \\
\text { LABORATORIAIS }\end{array}$ & CRITERIOS DE INCLUSÃO PARA DILI \\
\hline $\begin{array}{l}\text { GEARHART, M. } \\
\text { O., } 1994\end{array}$ & $\begin{array}{l}\text { AST, ALT, TB, AP, PT, } \\
\text { aPTT }\end{array}$ & AST=1556 U/L, ALT=62 U/L, TB=130 $\mu \mathrm{mol} / \mathrm{L} ; \mathrm{AP}=216 \mathrm{U} / \mathrm{L} ; \mathrm{PT}=40.5 \mathrm{~s} ; \mathrm{aPTT}=57.4 \mathrm{~s}$ \\
\hline $\begin{array}{l}\text { OZICK, L. A. et } \\
\text { al., } 1995\end{array}$ & ALT, AST & ALT ou AST > 200 U/L. \\
\hline $\begin{array}{l}\text { SCHABERG, T., } \\
1996\end{array}$ & GGT, AST, ALT & Enzimas hepáticas > $3 \mathrm{ULN}$ : GGT > $69 \mathrm{U} / \mathrm{L}^{-1}$; AST >54 U/L ${ }^{-1}$; ALT > $\left.60 \mathrm{U} / \mathrm{L}^{-1}\right)$. \\
\hline $\begin{array}{l}\text { BAGHERI, H. et } \\
\text { al., } 2000 .\end{array}$ & ALT, AP, GGT, TB & ALT $>2$ ULN ou AP > 1.5 e ALT ou GGT ou TB elevado. \\
\hline $\begin{array}{l}\text { FISCHER, M. A. } \\
\text { et al., } 2005\end{array}$ & AST, ALT & $\begin{array}{l}\text { ALT ou AST > } 3 \text { ULN. Para pacientes admitidos com níveis de transaminases (> 50\% ULN): uma } \\
\text { duplicação do nível de transaminases de admissão. }\end{array}$ \\
\hline $\begin{array}{l}\text { MEIER, Y. et al., } \\
2005\end{array}$ & ALT, AST, AP, CB & ALT $>2$ ULN ou CB $>2$ ULN ou AST, AP e TB $>2$ ULN. \\
\hline $\begin{array}{l}\text { MONTERO, A. } \\
\text { R. et al., } 2005\end{array}$ & ALT, AST, CB, TB, AP & ALT $>76 \mathrm{U} / \mathrm{L}$, ou CB $>0.6 \mathrm{mg} / \mathrm{mL}$, ou AST $>80 \mathrm{U} / \mathrm{L}, \mathrm{TB}>2 \mathrm{mg} / \mathrm{mL}$ e AP $>516 \mathrm{U} / \mathrm{L}$ \\
\hline $\begin{array}{l}\text { COCA, N. S. M. } \\
\text { at al, } 2010\end{array}$ & ALT, TB & ALT $>3$ ULN ou ALT > 3 ULN ou ALT > 3 ULN e TB > 2 ULN. \\
\hline $\begin{array}{l}\text { LIMA \& MELO, } \\
2012\end{array}$ & AST, ALT, TB & AST ou ALT > 3 ULN ou TB > 2 ULN \\
\hline $\begin{array}{l}\text { SIDERI, G. et al., } \\
2012\end{array}$ & $\begin{array}{l}\text { PT, PTTK, AST, ALT, AP, } \\
\text { TB }\end{array}$ & PT, PTTK, AST, ALT, AP, TB elevados \\
\hline XU, H.-M., 2012 & ALT, AP & 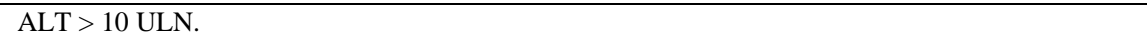 \\
\hline $\begin{array}{l}\text { LEE, T. et al., } \\
2013\end{array}$ & AST, ALT, AP, TB, PT & AST > $40 \mathrm{IU} / \mathrm{L}, \mathrm{ALT}>40 \mathrm{IU} / \mathrm{L}, \mathrm{AP}>120 \mathrm{IU} / \mathrm{L}, \mathrm{TB}>1.2 \mathrm{mg} / \mathrm{dL}$, ou PT $>1.3 \mathrm{INR}$ \\
\hline $\begin{array}{l}\text { BOHM, N. et al., } \\
2014\end{array}$ & ALT, TB & ALT > 2 ULN. TB $\geq 2.5 \mathrm{mg} / \mathrm{dL}$ (valor de linha de base $<1.3 \mathrm{mg} / \mathrm{dL}$ ). \\
\hline $\begin{array}{l}\text { CHALCO- } \\
\text { AGUATE, M. et } \\
\text { al., } 2014\end{array}$ & ALT & $\mathrm{ALT} \geq 3 \mathrm{ULN}$ \\
\hline $\begin{array}{l}\text { DOUROS, A. et } \\
\text { al., } 2014\end{array}$ & ALT, TB & ALT $>3$ ULN ou AST $>3$ ULN ou TB $>2 \mathrm{mg} \mathrm{dl}^{-1}$. \\
\hline $\begin{array}{l}\text { WOO, H. J. et al, } \\
2015\end{array}$ & $\begin{array}{l}\text { AST, ALP, DB, TB, AP, } \\
\text { GGT }\end{array}$ & ALT > 2 ULN; DB > 2 ULN, ou aumentos simultâneos em AST, ALP e TB, com um valor > 2 ULN \\
\hline $\begin{array}{l}\text { SCHNEEWEISS, } \\
\text { S. et al., } 2016\end{array}$ & AST, ALT & ALT ou AST > 5 ULN \\
\hline $\begin{array}{l}\text { FRIEDRICH, M.- } \\
\text { E. et al., } 2016\end{array}$ & AST, ALT, GGT, AP & AST ou ALT ou GGT ou AP > 5 ULN \\
\hline $\begin{array}{l}\text { ISA, S. E. et al., } \\
2016\end{array}$ & ALT, AST & $\begin{array}{l}\text { ALT > } 3 \text { ULN com características de hepatite sintomática ou ALT > } 5 \text { ULN na ausência de sintomas } \\
\text { de hepatotoxicidade. }\end{array}$ \\
\hline $\begin{array}{l}\text { SALOOJEE, A. } \\
\text { et al., } 2017\end{array}$ & TB, ALT & TB > $34.2 \mu \mathrm{mol} / \mathrm{L}(2 \mathrm{mg} / \mathrm{dL})$. Lesão hepática hipóxica: ALT > 20 ULN \\
\hline $\begin{array}{l}\text { VEKEMAN, F., } \\
2018\end{array}$ & AST, ALT, TB & $\begin{array}{l}\text { Grau 3: AST }>5 \text { ULN e }<20 \text { ULN; ALT }>5 \text { ULN e }<20 \text { ULN; TB }>3 \text { ULN e } \leq 10 \text { ULN. Grau 4: } \\
\text { AST } \geq 20 \text { ULN; ALT } \geq 20 \text { ULN; TB }>10 \text { ULN. Grau 5: morte. }\end{array}$ \\
\hline $\begin{array}{l}\text { EL KAHI, C. et } \\
\text { al., } 2018\end{array}$ & AST, ALT, GGT, AP & AST > 7 ULN; ALT > 7 ULN; AP=158 IU/L; GGT=201 UI/L; \\
\hline $\begin{array}{l}\text { FANG, W. C. et } \\
\text { al., } 2018\end{array}$ & ALT, AP, TB & $\begin{array}{l}\text { Leve: Elevado (ALT / AP), TB <2 ULN; Moderado: Elevado (ALT / AP), TB } \geq 2 \text { ULN } \\
\text { Grave: Elevado (ALT / AP), TB } \geq 2 \text { ULN e um dos seguintes: INR } \geq 1,5 \text {, ascite e/ou encefalopatia, }< \\
26 \text { semanas e ausência de cirrose subjacente / falência de outro órgão. Fatal: morte ou transplante de } \\
\text { fígado. }\end{array}$ \\
\hline $\begin{array}{l}\text { WANG, S. et al., } \\
2018\end{array}$ & AST, ALT, AP, TB & ALT ou AST > 5 ULN, ou AP ou TB > 2 ULN \\
\hline $\begin{array}{l}\text { CANO- } \\
\text { PANIAGUA, A. } \\
\text { et al., } 2019\end{array}$ & ALT, AP & ALT $>3$ ULN e/ou AP > 2 ULN \\
\hline $\begin{array}{l}\text { WORLAND, T. } \\
\text { et al., } 2020\end{array}$ & $\mathrm{ALT}, \mathrm{TB}, \mathrm{AP}$ & $\mathrm{TB}>200 \mu \mathrm{mol} / \mathrm{L}$ ou $\mathrm{ALT}>1,000 \mathrm{IU} / \mathrm{L}$ ou AP $>500 \mathrm{IU} / \mathrm{L}$ \\
\hline $\begin{array}{l}\text { ALI, N. et al, } \\
2020\end{array}$ & ALT & ALT > 5 ULN em indivíduos assintomáticos e ALT > 3 ULN em pacientes sintomáticos. \\
\hline
\end{tabular}

PT: tempo de protrombina; aPTT: tempo de tromboplastina parcial ativado; PTTK: tempo de protrombina com caulim; ALT: alanina aminotransferase; AST: aspartato aminotransferase; AP: fosfatase alcalina; TB: bilirrubina total; DB: bilirrubina direta; CB: bilirrubina conjugada; GGT: gama glutamil transpeptidase; ULN: limite superior do normal (foram usados valores normais específicos do laboratório); INR: razão normalizada internacional. Fonte: Gonçalves, et al. (2021).

A avaliação de causalidade por meio de algoritmos, tem por objetivo analisar a probabilidade de um determinado medicamento ser a causa de um efeito adverso, observado por meio da relação temporal entre a administração do medicamento e o aparecimento do evento, da exclusão de outros possíveis diagnósticos, ou seja, causas alternativas que possam explicar o caso, da existência de fundamentos na literatura científica para o medicamento suspeito, da reação do organismo ante a retirada 
do medicamento (melhora nos sinais e sintomas), bem como a piora nos sinais e sintomas com a reexposição ao medicamento (Teschke \& Andrade, 2016; Tan et al., 2018; Danan \& Teschke., 2016).

Existem alguns algoritmos específicos para a identificação da causalidade entre a ocorrência de lesão hepática e o uso de medicamento, tais como: RUCAM (Danan \& Teschke, 2016), Escala Maria \& Vitorino (Maria \& Victorino, 1997) e DDWJ (Digestive Disease Week Japan Scale) (Hanatani et al., 2014). Entretanto, vários autores apontam o algoritmo RUCAM como o padrão a ser seguido na identificação de DILI por sua sensibilidade e especificidade elevadas (Andrade et al., 2005; Björnsson et al., 2013; Hernandez et al., 2014; Chalasani et al, 2015; Danan \& Teschke, 2019;).

A lesão hepática suspeita de ser causada por drogas é definida pelos valores limites dos testes hepáticos, alanina aminotransferase (ALT) e/ou fosfatase alcalina (AP que vem do inglês alkaline phosphatase) (Danan \& Teschke, 2016). As atividades séricas consideradas relevantes são para ALT $\geq 5$ vezes o limite superior normal (ULN que vem do inglês upper limit of normal) e AP $\geq 2$ vezes o ULN, desde que AP seja de origem hepática (Danan, \& Teschke, 2016). Assim, os valores de ALT eliminam casos sem relevância clínica.

O RUCAM original foi o primeiro método de avaliação de causalidade a considerar os critérios de limiar, embora os valores inicialmente propostos para ALT fossem menores (Danan, \& Benichou, 1993) em comparação com os critérios atualmente usados (Danan, \& Teschke, 2016). Digno de nota, os pacientes com suspeita de DILI, que apresentam valores de testes hepáticos abaixo dos limiares, são considerados como não tendo lesão hepática clinicamente significativa, mas adaptação ou tolerância hepática (Teschke, 2019). Lamentavelmente, relatos de casos publicados sobre DILI ocasionalmente contêm casos sem mencionar os valores de ALT ou AP, excluindo-os como casos de DILI. Os valores de limiares atuais, utilizando o RUCAM atualizado, são geralmente bem aceitos internacionalmente, (Danan \& Teschke, 2016; Teschke, 2019; Teschke \& Danan, 2020; Wang, et al, 2018; Yang et al., 2019).

Além dos limites básicos relacionados ao RUCAM, considerar a bilirrubinemia era apenas um pequeno passo para prever um resultado mais sério de lesão hepática. No entanto, a bilirrubina não faz parte do algoritmo RUCAM de diagnóstico e não será considerada mais na análise atual. Da mesma forma, a histologia hepática não é incluída como um elemento no RUCAM, porque não há características típicas de DILI (Danan \& Benichou, 1993; Danan \& Teschke, 2016).

RUCAM também foi o primeiro método de avaliação de causalidade diferenciando padrões de lesão hepática, com base em testes hepáticos, sem a necessidade de histologia. Portanto, para determinar os tipos de lesão hepática, deve-se calcular o valor de R, que representa a razão de ALT dividido por AP, no início da lesão hepática. Para determinar lesão hepatocelular considera-se R > 5 e uma lesão hepática colestática / mista, R $\leq 5$ (Danan \& Benichou, 1993; Danan \& Teschke, 2016).

Nos 27 artigos, foram identificados relatos de uma grande variedade de medicamentos, como prováveis causas de DILI em pacientes hospitalizados. Para uma melhor visualização dos resultados, organizamos os medicamentos relatados de acordo com a Classificação Anatômica Terapêutica Química (ATC), que é utilizada internacionalmente para classificar as moléculas (substâncias) com ação terapêutica, sendo esta classificação adotada pela Organização Mundial de Saúde (World Health Organization, 2020).

De acordo com esta classificação, o grupo $\mathrm{J}$ corresponde aos medicamentos anti-infecciosos, $\mathrm{M}$ aos medicamentos que atuam no sistema músculo esquelético, $\mathrm{N}$ aos medicamentos que atuam no sistema nervoso central e $\mathrm{C}$ aos medicamentos que atuam no sistema cardiovascular. Entre os relatos de medicamentos encontrados, associados a DILI (Tabela 3), os de ocorrência mais frequente, de acordo com a classificação ATC, foram: 85\% (23) na classe J, 37\% (10) na classe M, 33,3\% (9) na classe N, e 33,3\% (9) na classe C. Encontrou-se também, em menor número, relatos de ocorrência de DILI com medicamentos antineoplásicos (Grupo L), antitrombóticos (Grupo B) e que atuam no trato alimentar e metabolismo (Grupo A). 
Tabela 3: Principais medicamentos relacionados à DILI em pacientes hospitalizados.

\begin{tabular}{|c|c|c|c|c|}
\hline $\begin{array}{c}\text { ATC } \\
\text { CLASSIFICAÇ̃̃OO }\end{array}$ & $\begin{array}{l}\text { CLASSE } \\
\text { FARMACOLÓG } \\
\text { ICA }\end{array}$ & MEDICAMENTOS & ARTIGOS & $\mathbf{N}$ \\
\hline \multirow{3}{*}{$\mathbf{J}$} & ANTIBIÓTICOS & $\begin{array}{l}\text { Amoxicilina/clavulanato, Cefalosporinas, } \\
\text { Imipenem, Sulfonamida, Lincomicina, Ciprofloxacina, } \\
\text { Cefminox, Ornidazol, Panipenem e Betamipron, Meropenem, } \\
\text { Cefoxitina, Quinolonas, Vancomicina, Macrolídeos, } \\
\text { Fluorquinolonas, Moxifloxacina, Piperacilina-Tazobactam, } \\
\text { Penicilinas, Nevirapina, Sulfametoxazol e Trimetoprim, } \\
\text { Azitromicina, Ácido Fusídico, Daptomicina, Lincomicina, } \\
\text { Metronidazol, Nitrofurantoína, Minociclina, Cefacloxacilina. }\end{array}$ & $\begin{array}{l}\text { MEIER, Y. et al., 2005; MONTERO, } \\
\text { A. R. et al., 2005; XU, H. M. et al., } \\
\text { 2012; LEE, T. et al., 2013; BOHM, } \\
\text { N. et al., 2014; DOUROS, A. et al., } \\
\text { 2015; WOO, H. J. et al, 2015; } \\
\text { SALOOJEE, A. et al., 2017; FANG, } \\
\text { W. C. et al., 2018; CANO- } \\
\text { PANIAGUA, A. et al., 2019; } \\
\text { WORLAND, T. et al., } 2020\end{array}$ & 11 \\
\hline & ANTIFÚNGICOS & $\begin{array}{l}\text { Fluconazol, Anfotericina B, Anfotericina B lipossomal, } \\
\text { Itraconazol, Micafungina, Anidulafungina, Caspofungina, }\end{array}$ & $\begin{array}{l}\text { GEARHART, M. O., 1994; } \\
\text { FISCHER, M. A. et al., 2005; } \\
\text { SIDERI, G. et al., 2012; } \\
\text { SCHNEEWEISS, et al., 2016; ISA, } \\
\text { S. E. et al., 2016; VEKEMAN, F., } \\
\text { 2018; MONTERO, A. R. et al., } \\
\text { 2005; FANG, W. C. et al., 2018 }\end{array}$ & 8 \\
\hline & $\begin{array}{l}\text { ANTITUBERCU } \\
\text { LOSTÁTICOS }\end{array}$ & Rifampicina, Pirazinamida, Isoniazida e Etambutol & $\begin{array}{l}\text { OZICK, L. A. et al., 1995; } \\
\text { SCHABERG, T., 1996; COCA, N. S. } \\
\text { M. at al, 2010; LIMA \& MELO, } \\
\text { 2012; LEE, T. et al., 2013; ISA, S. E. } \\
\text { et al., 2016; DOUROS, A. et al., } \\
\text { 2015; FANG, W. C. et al., 2018; } \\
\text { WANG, S. et al., 2018; CANO- } \\
\text { PANIAGUA, A. et al., 2019; ALI, N. } \\
\text { et al, 2020 }\end{array}$ & 11 \\
\hline $\mathbf{M}$ & $\begin{array}{l}\text { SISTEMA } \\
\text { MUSCULO- } \\
\text { ESQUELÉTICO }\end{array}$ & $\begin{array}{l}\begin{array}{l}\text { Cetoprofeno, } \\
\text { Baclofeno, }\end{array} \text { Alopurinol, } \\
\text { Talniflumato }\end{array}$ & $\begin{array}{l}\text { BAGHERI, H. et al., 2000; } \\
\text { MONTERO, A. R. et al., 2005; XU, } \\
\text { H.M. et al., 2012; LEE, T. et al., } \\
\text { 2013; DOUROS, A. et al., WOO, H. } \\
\text { J. et al, 2015; 2015; EL KAHI, C. et } \\
\text { al, 2018; FANG, W. C. et al., 2018; } \\
\text { CANO-PANIAGUA, A. et al., 2019; } \\
\text { WORLAND, T. et al., } 2020\end{array}$ & 10 \\
\hline \multirow{8}{*}{$\mathbf{N}$} & ANALGESICOS & Petidina, Morfina, Metamizol, Paracetamol & DOUROS, A. et al., 2015 & 1 \\
\hline & $\begin{array}{l}\text { ANTIDEPRESSI } \\
\text { VOS }\end{array}$ & 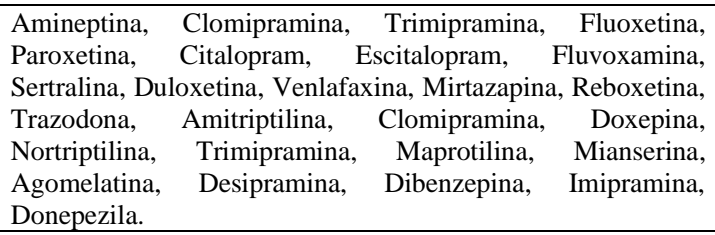 & $\begin{array}{l}\text { BAGHERI, H. et al., 2000; } \\
\text { MONTERO, A. R. et al., 2005; XU, } \\
\text { H.M. et al., 2012; WOO, H. J. et al, } \\
\text { 2015; FRIEDRICH, M. et al., 2016; }\end{array}$ & 5 \\
\hline & $\begin{array}{l}\text { ANTIPSICÓTIC } \\
\text { OS }\end{array}$ & $\begin{array}{l}\text { Clorpromazina, Haloperidol, Levomepromazina, Olanzapina, } \\
\text { Clozapina, Quetiapina }\end{array}$ & $\begin{array}{l}\text { BAGHERI, H. et al., 2000.; } \\
\text { MONTERO, A. R. et al., 2005; XU, } \\
\text { H. M. et al, 2012; DOUROS, A. et } \\
\text { al., 2015; FANG, W. C. et al., 2018 }\end{array}$ & 5 \\
\hline & ANXIOLÍTICOS & $\begin{array}{l}\text { Clorazepate, Lorazepam, Diazepam, Tetrazepam, Midazolam, } \\
\text { Hidroxizina }\end{array}$ & $\begin{array}{l}\text { BAGHERI, H. et al., 2000; } \\
\text { MONTERO, A. R. et al., 2005; } \\
\text { DOUROS, A. et al., } 2015\end{array}$ & 3 \\
\hline & $\begin{array}{l}\text { ANTIEPILÉPTIC } \\
\text { OS }\end{array}$ & $\begin{array}{l}\text { Clonazepam, Ácido valpróico, Carbamazepina, Lamotrigina, } \\
\text { Fenitoína, Pregabalina, Gabapentina }\end{array}$ & $\begin{array}{l}\text { BAGHERI, H. et al., 2000, } \\
\text { MONTERO, A. R. et al., 2005; LEE, } \\
\text { T. et al., 2013; DOUROS, A. et al., } \\
\text { 2015; WOO, H. J. et al, 2015; } \\
\text { FANG, W. C. et al., 2018; CANO- } \\
\text { PANIAGUA, A. et al., 2019 }\end{array}$ & 7 \\
\hline & $\begin{array}{l}\text { ANTI- } \\
\text { PARKINSONIA } \\
\text { NOS } \\
\end{array}$ & Levodopa, Biperideno, Amantadina, & $\begin{array}{l}\text { MONTERO, A. R. et al., 2005; } \\
\text { DOUROS, A. et al., 2015; FANG, } \\
\text { W. C. et al., 2018 }\end{array}$ & 3 \\
\hline & ANESTÉSICOS & Etomidato, Bupivacaína, Fentanil & DOUROS, A. et al., 2015 & 1 \\
\hline & $\begin{array}{l}\text { HIPNÓTICOS E } \\
\text { SEDATIVOS }\end{array}$ & Zolpidem & DOUROS, A. et al., 2015 & 1 \\
\hline $\mathbf{C}$ & $\begin{array}{l}\text { SISTEMA } \\
\text { CARDIOVASCU } \\
\text { LAR }\end{array}$ & $\begin{array}{l}\text { Quinapril, Fenofibrato, Atorvastatina, Captopril, Verapamil, } \\
\text { Pravastatina, Amiodarona, Cinepazida, Telmisartan, } \\
\text { Norepinefrina, Dobutamina, Olmesartan, } \\
\begin{array}{ll}\text { Furosemida, Telmisartan, Amlodipina } & \text { Enalapril; }\end{array}\end{array}$ & $\begin{array}{l}\text { BAGHERI, H. et al., 2000; } \\
\text { MONTERO, A. R. et al., 2005; XU, } \\
\text { H. M.et al, 2012; DOUROS, A. et } \\
\text { al., 2015; WOO, H. J. et al, 2015; } \\
\text { ISA, S. E. et al., 2016; SALOOJEE, } \\
\text { A. et al., 2017; FANG, W. C. et al., } \\
\text { 2018; WORLAND, T. et al., 2020 }\end{array}$ & 9 \\
\hline
\end{tabular}




\begin{tabular}{|c|c|c|c|c|}
\hline $\mathbf{L}$ & $\begin{array}{l}\text { AGENTES } \\
\text { ANTINEOPLÁST } \\
\text { ICOS }\end{array}$ & 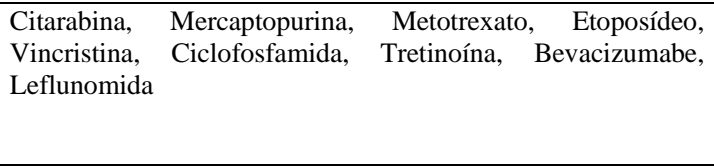 & $\begin{array}{l}\text { MEIER, Y. at al., 2005; MONTERO, } \\
\text { A. R. et al., 2005; XU, H.M. et al, } \\
\text { 2012; LEE, T. et al., 2013; FANG, } \\
\text { W. C. et al., 2018; WORLAND, T. et } \\
\text { al., } 2020\end{array}$ & 6 \\
\hline B & $\begin{array}{l}\text { AGENTES } \\
\text { ANTITROMBÓT } \\
\text { ICOS }\end{array}$ & $\begin{array}{lccc}\text { Fluindiona, Heparina não } & \text { fracionada, } & \text { Dalteparina, } \\
\text { Clopidogrel, } & \text { Ticlopidina, } & \text { Heparina, } & \text { Enoxaparina, } \\
\text { Certoparina, Rivaroxabana, Ácido acetilsalicílico }\end{array}$ & $\begin{array}{l}\text { BAGHERI, H. et al., 2000; MEIER, } \\
\text { Y. at al., 2005; MONTERO, A. R. et } \\
\text { al., 2005; DOUROS, A. et al., 2015; } \\
\text { EL KAHI, C. et al, 2018; CANO- } \\
\text { PANIAGUA, A. et al., 2019 }\end{array}$ & 6 \\
\hline $\mathbf{A}$ & $\begin{array}{l}\text { TRATO } \\
\text { ALIMENTAR E } \\
\text { METABOLISMO }\end{array}$ & $\begin{array}{l}\text { Sulfassalazina, Ranitidina, Omeprazol, } \\
\text { Metoclopramida, Dantoprazol, } \\
\text { Multivitaminas, Potássio, }\end{array}$ & $\begin{array}{l}\text { BAGHERI, H. et al., 2000; } \\
\text { MONTERO, A. R. et al., 2005; XU, } \\
\text { H. M. et al, 2012; DOUROS, A. et } \\
\text { al., 2015; FANG, W. C. et al., 2018 }\end{array}$ & 5 \\
\hline
\end{tabular}

Fonte: Gonçalves, et al. (2021).

Nesta pesquisa verificamos que o grupo $\mathrm{J}$, que abrange diferentes fármacos anti-infecciosos, foi o que teve maior número de relatos de DILI, sendo 47,8\% (11) de antibióticos, 47,8\% (11) de antituberculostáticos e 34,7 \% (8) de antifúngicos. Dos antibióticos relatados, os de ocorrência mais frequente, representando 51,4\% foi o grupo dos Beta-lactâmicos (18), destacando-se a Amoxacilina/clavulanato com 50\% (9).

Em vários estudos prospectivos e retrospectivos sobre DILI, os antibióticos foram os mais relatados como causa de lesão hepática (Björnsson \& Olsson, 2005; Andrade et al., 2005; De Valle et al., 2006; Chalasani et al, 2008; Chalasani et al, 2015; Björnsson, 2016). Não está claro por que os antibióticos são tão propensos a causar lesões hepáticas em comparação com outros fármacos. No entanto, os antibióticos são comumente prescritos e amplamente utilizados na prática clínica.

A DILI devido à exposição a Amoxicilina-clavulanato (AC) é observada com relativa frequência. A Rede de Lesões Hepáticas Induzidas por Drogas dos EUA (DILIN) (Chalasani et al, 2008) e o Registro Espanhol de DILI (Andrade et al, 2005) identificaram a AC como a causa mais comum de lesão hepática. A incidência de AC-DILI foi determinada em um estudo prospectivo de 2 anos na Islândia, sendo que dos 35.000 pacientes em estudo, foram identificados 15 casos de AC-DILI, correspondendo a 1 em 2.350 pacientes (43 casos por 100.000) (Björnsson et al., 2013). Se assumirmos a generalização para os EUA, onde mais de 70 milhões de americanos são tratados a cada ano com AC, isso sugeriria que poderia haver anualmente cerca de 30.000 de casos de DILI devido a esta associação de fármacos anualmente nos EUA (Fontana, 2014).

Da classe dos antifúngicos destacou-se o Fluconazol com 62,5\% dos relatos de DILI. Todos os agentes antifúngicos, incluindo formulações de Anfotericina B, equinocandinas e os triazóis, podem causar toxicidade hepática que varia de anormalidades leves e assintomáticas à lesão hepática substancial e insuficiência hepática fulminante (Kyriakidis et al, 2017).

Quanto aos antituberculostáticos, o tratamento citado em 11 artigos (47,8\%) incluía Rifampicina, Izoniazida e Pirazinamida e apenas 2 relataram o uso de Etambutol. A DILI é uma reação adversa importante do tratamento anti-TB, levando à não adesão, falha do tratamento ou desenvolvimento de resistência aos medicamentos (Tang, et al., 2012). Globalmente, a DILI anti-TB é relatada em $2 \%$ a $28 \%$ dos pacientes de acordo com várias definições, populações de estudo e regimes de tratamento (Shang et al., 2011).

Dos 12 relatos dos medicamentos do grupo $\mathrm{M}$, medicamentos que atuam no sistema musculoesquelético, distinguiu-se com o percentual de 16\% para cada fármaco: Diclofenaco de sódio e Alopurinol.

Houve uma variedade muito grande de relatos de medicamentos do grupo $\mathrm{N}$, sobressaindo os antiepilépticos com 27,3\%. Dentre os 11 relatos, destacou-se o Ácido Valpróico (27,3\%), Carbamazepina (18,8\%) e Fenitoína (18,8\%).

Foram relatados vários medicamentos que atuam no sistema cardiovascular, Grupo C, tais como, Artovastatina, Amiodarona, Enalapril e Telmisartan, correspondendo a 10,5 \% cada.

Em um estudo, os casos de DILI com base no RUCAM foram recuperados de 15 relatórios publicados por seis bancos de dados internacionais de registros de DILI e três grandes centros médicos, que forneceram 3312 casos. No geral, 48 
medicamentos com o maior número de casos de DILI foram listados. Entre os 10 principais medicamentos implicados em causar DILI estavam, em ordem decrescente: Amoxicilina-clavulanato, Flucloxacilina, Atorvastatina, Dissulfiram, Diclofenaco, Sinvastatina, Carbamazepina, Ibuprofeno, Eritromicina e esteroides anabolizantes. Para esses 10 medicamentos, os respectivos números de casos DILI foram mais altos para Amoxicilina-clavulanato ( $\mathrm{n}=333$ ) (Teschke, 2018). Esta classificação de medicamentos fornece apenas uma visão geral, mas não substitui as considerações sobre as especificidades de países individuais com um espectro de doenças diferentes, incluindo ou excluindo, por exemplo, tuberculose, causando variabilidades no uso de drogas e DILI associada (Björnsson, 2016; Teschke, 2018).

Na série publicada, de coorte DILIN (Drug-Induced Liver Injury Network) nos EUA (2004-2013), os anti-infecciosos, incluindo antibióticos e antituberculostáticos, correspondem aproximadamente a $46 \%$ de todos os casos de DILI (Chalasani et al., 2015). Adicionalmente, em um estudo de base populacional realizado na França, a incidência de DILI foi de aproximadamente 14 casos por 100.000 habitantes e $25 \%$ estava associado a fármacos anti-infecciosos (Sgro et al., 2002). Também, em uma revisão crítica de casos brasileiros de DILI, os anticonvulsivantes foram os mais relatados, sendo o mais frequente o Ácido Valpróico (Becker et al, 2019).

Em geral, o número de pacientes hospitalizados por outras causas, que desenvolveram DILI durante a hospitalização, é maior do que o número de pacientes internados inicialmente por causa da DILI (Becker et al., 2019). Esses resultados sugerem que pode ser importante monitorar cuidadosamente a função hepática dos pacientes e também coletar mais dados sobre drogas potencialmente hepatotóxicas usadas em hospitais. Além disso, o uso de RUCAM sempre que houver suspeita de DILI, deve ser estimulado (Danan \& Teschke, 2016).

\section{Considerações Finais}

Esta revisão identificou relatos de uma grande variedade de medicamentos responsáveis pela ocorrência de DILI em pacientes hospitalizados. Os relatos de medicamentos associados a DILI, de acordo com a classificação ATC, foram: 85\% (23) de fármacos do grupo J, 37\% (10) do grupo $\mathrm{M}, 33,3 \%$ (9) do grupo N, e 33,3\% (9) do grupo C. O grupo J, que abrange fármacos antiinfecciosos, foi o que teve maior número de relatos, sendo 47,8\% (11) de antibióticos, 47,8\% (11) de antituberculostáticos e 34,7 \% (8) de antifúngicos. Destes, destacaram-se os Beta-lactâmicos, em especial a AmoxacilinaClavulanto, Rifampicina, Isoniazida, Pirazinamida e Fluconazol.

Considerando que o desfecho da DILI pode ser grave, a detecção precoce e o manejo da hepatotoxicidade são necessários, bem como o monitoramento farmacoterapêutico e dos exames laboratoriais dos pacientes. Além disso, a aplicação do algoritmo RUCAM na prática clínica deve ser mais divulgada e utilizada no manejo terapêutico.

Por fim, novas pesquisas que visem ampliar o conhecimento de reações adversas a medicamentos pelos profissionais da área da saúde, permitirão a implementação de ações que podem contribuir para a detecção precoce da DILI, aumentando a segurança da terapêutica.

\section{Agradecimentos}

O Presente trabalho foi realizado com apoio da Coordenação de Aperfeiçoamento de Pessoal de Nível Superior Brasil (Capes) - Código de Financiamento - 001

\section{Referências}

Ali, N., Gupta, N., \& Saravu, K. (2020). Malnutrition as an important risk factor for drug-induced liver injury in patients on anti-tubercular therapy: an experience from a tertiary care center in South India. Drug Discoveries \& Therapeutics, 14(3), 135-138. https://doi.org/10.5582/ddt.2020.03029 
Andrade, R. J., Lucena, M. I, Fernandez, M. C., Pelaez, G., Pachkoria, K., García-Ruiz, E., García-Munõz, B., González-Grande, R., Pizzaro, A., Durán, J. A., Jiménez, M., Rodrigo, L., Romero-Gomez, M., Navarro, J. M., Planas, R., Costa, J., Borras, A., Soler, A., Salmerón, J., \& Martin-Vivaldi, R. (2005) Drug-induced liver injury: an analysis of 461 incidences submitted to the Spanish registry over a 10-year period. Gastroenterology, $129,512-521$.

Bagheri, H., Michel, F., Lapeyre-Mestre, M., Lagier, E., Cambus, J. P., Valdiguie, P., \& Montastruc, J. L. (2000). Detection and incidence of drug-induced liver injuries in hospital: a prospective analysis from laboratory signals. Br J Clin Pharmacol, 50(5), 479-484. http://doi.org/10.1046/j.1365-2125.2000.00282x

Bessone, F., Hernandez, N., Mendizabal, M., Sanchez, A., Paraná, R., Arrese, M., Tagle, M., Girala, M., Lizarzabal, M., Carrera, E., Brahm, J., Contreras, F., Mendez-Sanchez, N., Santos, G., Nunes, V., Medina-Caliz, I., Parra-Martinez, C., Sanz-Villanueva, L., Lucena, M. I., \& Andrade, R. J. (2019). When the Creation of a Consortium Provides Useful Answers: Experience of The Latin American DILI Network (LATINDILIN). Clinical Liver Disease, 13 (2), 51-57.

Becker, M. W., Lunardelli, M. J. M., Tovo C. V., \& Blatt, C. R. (2019). Drug and herb-induced liver injury: A critical review of Brazilian cases with proposals for the improvement of causality assessment using RUCAM. Annals of Hepatology, 18(5), 742-750. https://doi.org/10.1016/j.aohep.2019.03.010

Björnsson, E, \& Olsson, R. (2005). Outcome and prognostic markers in severe drug-induced liver disease. Hepatology, 42, 481-489.

Björnsson, E. (2010). Review article: drug-induced liver injury in clinical practice. Aliment Pharmacol Ther, 32(3), 3-13.

Björnsson, E. S., Bergmann, O. M., Björnsson, H. K., Kvaran, R. B., \& Olafsson, S. (2013). Incidence, presentation, and outcomes in patients with druginduced liver injury in the general population of Iceland. Gastroenterology, 144(7), 1419-1425. https://doi.org/10.1053/j.gastro.2013.02.006

Björnsson, E. S. (2016). Hepatotoxicity by Drugs: The Most Common Implicated Agents. Int. J. Mol. Sci., 17(2), 224. https://doi.org/10.3390/ijms17020224

Bohm, N., Makowski, C., Machado, M., Davie, A., Seabrook, N., Wheless, L, Bevill, B., Clark, B., \& Kyle, T. G. (2014). Case Report and Cohort Analysis of Drug-Induced Liver Injury Associated with Daptomycin. Antimicrobial Agents and Chemotherapy, 58(8), $4902-4903$.

Chalasani, N., Fontana, R. J., Bonkovsky, H. L, Watkins, P. B., Davern, T., Serrano, J., Yang, H., Rochon, J., \& for the Drug Induced Liver Injury Network (DILIN). (2008). Causes, Clinical Features, and Outcomes From a Prospective Study of Drug-Induced Liver Injury in the United States. Gastroenterology, 135(6), 1924-1934.

Chalasani, N., Bonkovsky, H. L., Fontana, R., Lee, W., Stolz, A, Talwalkar, J., Reddy, K. R., Watkins, P. B., Navarro, V., Barnhart, H., Gu, J. \& Serrano, J. (2015). Features and outcomes of 899 patients with drug-induced liver injury: the DILIN Prospective Study. Gastroenterology, 148, $1340-1352$.

Coca, N. S. M., Oliveira, M. S., Voieta, I, Antunes, C. M. de F., \& Lambertucci, J. R. (2010). Antituberculosis drug-induced hepatotoxicity: a comparison between patients with and without human immunodeficiency virus seropositivity. Revista da Sociedade Brasileira de Medicina Tropical, 43(6), 624-628.

Cano-Paniagua, A., Amariles, P, Ângulo, N., \& Restrepo-Garaya, M. (2019). Epidemiology of drug-induced liver injury in a University Hospital from Colombia: Updated RUCAM being used for prospective causality assessment. Annals of Hepatology, 18, $501-507$.

Chalco-Aguate, M., Sáenz-Anduaga, E., Oré-Mora, M., Quiñones-Velarde, J., Chanco-Ramírez, G., \& Anco-Gallegos, K. (2014). Falla terapéutica y hepatotoxicidad de los esquemas de tratamiento endovenoso e intramuscular con estibogluconato de sodio en pacientes con leishmaniasis cutánea. Dermatol Peru, 24(3), 153-158.

Chalasani, N, Bonkovsky, H.L., Fontana, R., Lee W, Stolz, A, Talwalkar, J., Reddy, K. R., Watkins, P. B., Navarro, V., Barnhar, H., Gu, J., \& Serrano, J. (2015). Features and outcomes of 899 patients with drug-induced liver injury: the DILIN prospective study. Gastroenterology, 148(7), 1340-1352. https://doi.org/10.1053/j.gastro.2015.03.006

Chitturi S., \& Farrell G.C. (2011). Drug-induced liver disease. In: E. R. Schiff, W. C. Maddrey, \& M. F. Sorrell, Schiff's Diseases of the Liver (11th Edition), 703-783. USA: Wiley.

Danan, G., \& Benichou, C. (1993). Causality assessment of adverse reactions to drugs-I. A novel method based on the conclusions of international consensus meetings: Application to drug-induced liver injuries. J. Clin. Epidemiol., 46(11), 1323-1330.

Danan, G., \& Teschke, R. (2016). RUCAM in Drug and Herb Induced Liver Injury: The Update. Int. J. Mol. Sci., 17(1), 14. https://doi.org/10.3390/ijms17010014

Danan, G, \& Teschke, R. (2019). Roussel Uclaf Causality Assessment Method for Drug-Induced Liver Injury: Present and Future. Front. Pharmacol., 10, 853. https://doi.org/10.3389/fphar.2019.00853

De Valle, M. B., Klinteberg, V., Alem, N., Olsson, R., \& Björnsson, E. (2006). Drug-induced liver injury in a Swedish University hospital outpatient hepatology clinic. Aliment Pharmacol Ther., 24, 1187-1195.

Douros, A., Bronder, E., Andersohn, F., Klimpel, A., Thomae, M., Sarganas, G., Kreutz, R., \& Garbe, E. (2014). Drug-induced liver injury: results from the hospital-based Berlin Case-Control Surveillance Study. Br J Clin Pharmacol, 79(6), 988-999.

El Kahi, C., Martinet, V., Praet, J. P., \& Pepersack, T. (2018). Un cas rare d’hépatotoxicité en gériatrie. Revue médicale de Bruxelles, 39(3),164-165.

Fang, W. C., Adler, N. R., Graudins, L. V., Goldblatt, C., Goh, M. S. Y., Roberts, S. K., Trubiano, J. A., \& Aung, A. K. (2018). Drug-induced liver injury is frequently associated with severe cutaneous adverse drug reactions: experience from two Australian tertiary hospitals. Internal Medicine Journal, 48(5), 549555. https://doi.org/10.1111/imj.13734

Fischer, M. A., Winkelmayer, W. C., Rubin, R. H., \& Avorn, J. (2005). The Hepatotoxicity of Antifungal Medications in Bone Marrow Transplant Recipients. Clinical Infectious Diseases, 41, 301-307. 
Friedrich, M-E., Akimova, E., Huf, W., Konstantinidis, A., Papageorgiou, K., Winkler, D, Toto, S., Greil, W., Grohmann, R., \& Kasper, S. (2016). DrugInduced Liver Injury during Antidepressant Treatment: Results of AMSP, a Drug Surveillance Program. International Journal of Neuropsychopharmacology, 19(4), 1-9. http:// doi:10.1093//injp/pyv126

Fontana, R. J., Hayashi, P. H., Gu, J., Reddy, K. R., Barnhart, H, Watkins, P. B., Serrano, J., Lee, W. M., Chalasani, N., Stolz, A., Davern, T., \& Talwakar, J. A. (2014). Idiosyncratic drug-induced liver injury is associated with substantial morbidity and mortality within 6 months from onset. Gastroenterology, 147(1), 96-108. https://doi.org/10.1053/j.gastro.2014.03.045

Fontana, R. J. (2014). Pathogenesis of idiosyncratic drug-induced liver injury and clinical perspectives. Gastroenterology, 146, 914-928.

Galvão, C. M. (2006). Níveis de evidência [editorial]. Acta Paul Enferm, 19(2).

Ganger, D. R., Rule, J., Rakela, J., Bass, N., Reuben, A., Stravitz, R. T., Sussman, N., Larson, A. M., James, Laura, Chiu, Charles, \& Lee, W. M. (2018). Acute liver failure of indeterminate etiology: a comprehensive systematic approach by an expert committee to establish causality. Am J Gastroenterol., 113(9), 1319.

García-Cortés, M., Stephens, C., Lucena, M. I., Fernández-Castañer, A., \& Andrade, R. J. (2011). Causality assessment methods in drug induced liver injury: strengths and weaknesses. J Hepatol., 55, 683-691.

Goldkind, L, \& Laine, L. (2006). A systematic review of NSAIDs withdrawn from the market due to hepatotoxicity: lessons learned from the bromfenac experience. Pharmacoepidemiol Drug Saf, 15, 213-220.

Gearhart, M. O. (1994). Worsening of Liver Function with Fluconazole and Review of azole antifungal Hepatotoxicity. The Annals of Pharmacotherapy, 28, $1177-1181$.

Hanatani, T., Sai1, K., Tohkin, M., Segawa, K., Kimura, M., Hori, K., Kawakami, J., \& Saito, Y. (2014). A detection algorithm for drug-induced liver injury in medical information databases using the Japanese diagnostic scale and its comparison with the council for international organizations of medical sciences/the roussel uclaf causality assessment method scale. Pharmacoepidemiology and Drug Safety, 23 (9), 984-988.

Hernandez, N., Bessone, F., Sanchez, A., Di Pace, M., Brahm, J., Zapata, R., Chirino, R. A., Dávalos, M., Méndez-Sánchez, N., Arrese, M., Schinoni, M. Lucena, M. I., \& Andrade, R. J. (2014). Profile of idiosyncratic drug induced liver injury in Latin America: an analysis of published reports. Ann Hepatol., 13, 231-239.

Isa, S. E., Ebonyi, A. O., Shehu, N. Y., Idoko, P., Anejo-Okopi, J. A., Simji, G., Odesanya, R. U., Abah, I. O., \& Jimoh, H. O. (2016). Antituberculosis drugs and hepatotoxicity among hospitalized patients in Jos, Nigeria. International Journal of Mycobacteriology, 5(1), 21-26. http://doi.org/ $10.1016 /$ j.jimyco.2015.10.001

Jee, A., Sernoskie, S. C., \& Uetrecht, J. (2021). Idiosyncratic Drug-Induced Liver Injury: Mechanistic and Clinical Challenges. Int. J. Mol. Sci., $22,2954$.

Kyriakidis, I., Tragiannidis, A., Munchen, S., \& Groll, A. H.(2017). Clinical hepatotoxicity associated with antifungal agentes. Expert Opin Drug Saf., 16 (2), $149-165$.

Kullak-Ublick, G. A., Andrade, R. J., Merz, M., End, P., Benesic, A., Gerbes, A. L., \& Aithal, G. P. (2017). Drug-induced liver injury: recent advances in diagnosis and risk assessment. Gut, 66(6), 1154-1164. http://dx.doi.org/10.1136/gutjnl-2016-313369

Larrey, D. (2002). Epidemiology and individual susceptibility to adverse drug reactions affecting the liver. Semin Liver Dis., 22, 145-155.

Lee, W. M. (2013). Drug-induced acute liver failure. Clin Liver Dis, 17(4), 575-586. https://doi.org/10.1016/j.cld.2013.07.001

Lee, T., Lee, Y. S., Yoon, S-Y., Kim, S., Bae, Y-J., Kwon, H-S., Cho, Y., S., Moon, H-B., \& Kim, T-B. (2013). Characteristics of liver injury in drug-induced systemic hypersensitivity reactions. J Am Acad Dermatol, 69(3), 407-15. http://doi.org/10.1016/j.jaad.2013.03.024

Lima, M. F. S., \& Melo, H. R. L. (2012). Hepatotoxicity induced by antituberculosis drugs among patients coinfected with HIV and tuberculosis. Cadernos de Saúde Pública, 28(4), 698-708.

Maria, V. A., \& Victorino, R. M. (1997). Development and validation of a clinical scale for the diagnosis of drug-induced hepatitis. Hepatology, 26(3), 664669.

Mendes, K. D. S., Silveira, R. C. C. P., \& Galvão, C. M. (2008). Revisão integrativa: método de pesquisa para a incorporação de evidências na saúde e na enfermagem. Texto \& Contexto - Enfermagem, 17(4), 758-764.

Meier, Y., Cavallaro, M., Roos, M., Pauli-Magnus, C., Folkers, G., Meier, P. J., \& Fattinger, K. (2005). Incidence of drug-i'nduced liver injury in medical inpatients. Eur J Clin Pharmacol, 61, 135-143.

Moher, D., Liberati, A., Tetzlaff, J., Altman, D. G., \& The PRISMA Group. (2019). Preferred Reporting Items for Systematic Reviews and Meta-Analyses: The PRISMA Statement. Epidemiol. PLOS Medicine, 6(7): e1000097. https://doi.org/10.1371/journal.pmed.1000097

Montero, A. R., Durán Quintana, J. A., Jiménez Sáenz, M., \& Abadín Delgado, J. A. (2005). A strategy to improve the detection of drug-induced hepatotoxicity. Rev Esp Enferm Dig, 97(3), 155-160.

Ozick. L.A., Jacob, L., Comer, G. M., Lee, T. P., Ben-Zvi, J. Donelson, S. S., \& Felton, C. P. (1995). Hepatotoxicity from Isoniazid and Rifampin in InnerCity AIDS Patients. The American Journal of Gastroenterology, 90(11), 1978-80.

Ostapowicz G., Fontana, R. J., Schiodt, F. V., Larson, A., Davern, T. J., Han, S. H. B., McCashland, T. M., Shakil, A. O., Hay, J. E., Hynan, L., Crippin, J. S., Blei, A. T., Samuel, G., Reisch, J., \& Lee, W. M. (2002). Results of a prospective study of acute liver failure at 17 tertiary care centers in the United States. Ann Intern Med., 137(12), 947-54. https://doi.org/10.7326/0003-4819-137-12-200212170-00007 
Pereira, A. S., Shitsuka, D. M., Parreira, F. J. \& Shitsuka, R. (2018). Metodologia da pesquisa científica. [e-book]. Santa Maria. Ed. UAB/NTE/UFSM. https://repositorio.ufsm.br/bitstream/handle/1/15824/Lic_Computacao_Metodologia-Pesquisa-Cientifica.pdf?sequence=1

Saloojee, A., Skinner, D. L., Loots, E., Hardcastle, T. C., \& Muckart, D. J. J. (2017). Hepatic dysfunction: A common occurrence in severely injured patients. Injury Int. J. Care Injured, 48(1), 127-132. https://doi.org/10.1016/j.injury.2016.08.017

Santos, C. M. C., Pimenta, C. A. M., \& Nobre, M. R. C. (2007). The PICO strategy for the research question construction and evidence search. Revista LatinoAmericana de Enfermagem, 15(3), 508-511. https://doi.org/10.1590/S0104-11692007000300023

Santos, G., Figueira, E. R. R., D’Albuquerque, L. A. C., Lisboa, P. B., Almeida, M. D., Filgueira, N. A., Boinf, I., Porta, G., Alves da Silva, R. de C. M., Vianai, C. F. G., Fariaj, L. C., Alvares-da-Silva, M. R., Pereira de Moraes, A. C., Morsoletto, D. B. G., Codes, L., \& Paraná, R. (2021). Evaluation of druginduced liver injury as etiology for acute liver failure in Brazil. Annals of Hepatology, 23, 100310. https://doi.org/10.1016/j.aohep.2021.100310

Schaberg, T., Rebhan, K., \& Lode, H. (1996). Risk factors for side-effects of isoniazid, rifampin and pyrazinamide in patients hospitalized for pulmonary tuberculosis. Eur Respir J, 9, 2026-2030.

Schneeweiss, S., Carver, P. L., Datta, K., Galar, A., Johnson, M. D., Johnson, M. G., Marty, F. M., Nagel, J., Najdzinowicz, M., Saul, M., Shoham, S., Silveira, F. P., Varughese, C. A., Wilck, M., Weatherby, L., Auton, T., \& Walker, A. M. (2016). Short-term risk of liver and renal injury in hospitalized patients using micafungin: a multicentre cohort study. J Antimicrob Chemother, 71(10), 2938-44.

Sgro, C., Clinard, F., Ouazir, K., Chanay, H., Allard, C. Guilleminet, C., Lenoir, C., Lemoine, A., \& Hillon, P. (2002). Incidence of drug-induced hepatic injuries: a French population-based study. Hepatology, 36(2), 451-455. https://doi.org/10.1053/jhep.2002.34857

Shang, P., Xia, Y., Liu, F., Wang, X., Yuan, Y., Hu, D., Tu, D., Chen, Y., Deng, P., Cheng, S., Zhou, L., Ma, Y., Zhu, L., Gao, W., Wang, H., Chen, D., Yang, L., He, P., Wu, S., Tang, S., Lv, X., Shu, Z., Zhang, Y., Yang, Z., Chen, Y., Li, N., Sun, F., Li, X., He, Y., Garner, P., \& Zhan, S. (2011). Incidence, clinical features and impact on antituberculosis treatment of anti-tuberculosis drug-induced liver injury (ATLI) in China. PLOS One, 6, e21836.

Shen, T., Liu, Y., Shang, J., Xie, Q., Li, J., Yan M, Xu, J., Niu, J., Liu, J., Watkins, P. B., Aithal, G. P., Andrade, R. J., Dou, X., Yao, L., Lv, F., Wang, Q., Li, Y., Zhou, X., Zhang, Y., Zong, P., Wan, B., Zou, Z.., Yang, D., Nie, Y., Li, D., Wang, Y., Han, X., Zhuang, H., Mao, Y., \& Chen, C. (2019). Incidence and etiology of drug-induced liver injury in Mainland China. Gastroenterology, 156(8), 2230-2241. https://doi.org/10.1053/j.gastro.2019.02.002

Sideri, G, Falagas, M. E., Grigoriou, M., Vouloumanou, E. K., Papadatos, J. H., Lebessi, E., \& Kafetzis, D. A. (2012). Liposomal amphotericin B in critically ill paediatric patients. Journal of Clinical Pharmacy and Therapeutics, 37, 291-295.

Sousa, L. M. M. de, Marques-Vieira, C. M., Severino, S. S. P., \& Antunes, A. V. (2017). A metodologia da revisão integrativa da literatura em enfermagem. Revista Investigação em Enfermagem, 21, 17-26.

Souza, M. T., Silva, M. D., \& Carvalho, R. (2010). Revisão integrativa: o que é e como fazer. Einstein (São Paulo), 8(1), $102-106$.

Tang, S. W., Lv, X. Z., Zhang, Y., Wu, S. S., Yang, Z. R., Xia, Y. Y., Tu, D. H., Deng. P. Y., Ma, Y., Chen, D. F., \& Zhan, F. Y. (2012). CYP2E1, GSTM1 and GSTT1 genetic polymorphism and susceptibility to antituberculosis drug-induced hepatotoxicity: a nested case-control study. $J$ Clin Pharm Ther.,37, 588-593.

Tan, E. H., Low, E. X. S., Dan, Y. Y., \& Tai, B. C. (2018). Systematic review and meta-analysis of algorithms used to identify drug-induced liver injury (DILI) in health record databases. Liver International, 38, 742-753.

Teschke, R., \& Andrade, R. J. (2016). Drug, Herb, and Dietary Supplement Hepatotoxicity. Int. J. Mol. Sci., 17(9), 1488.

Teschke, R. (2018). Review. Top-ranking drugs out of 3312 drug-induced liver injury cases evaluated by the Roussel Uclaf Causality Assessment Method. Exp. Opin. Drug Metab. Toxicol. 14, 1169-1187. http://doi.org/10.1080/17425255.2018.1539077

Teschke, R. (2019), Idiosyncratic DILI: Analysis of 46,266 cases assessed for causality by RUCAM and published from 2014 to early 2019. Front. Pharmacol., 10, 730 .

Teschke, R., \& Danan, G. (2020). Worldwide Use of RUCAM for Causality Assessment in 81,856 Idiosyncratic DILI and 14,029 HILI Cases Published 1993-Mid 2020: A Comprehensive Analysis. Medicines, 7, 62.

Vekeman, F., Weiss, L., Aram, J., Ionescu-Ittu, R., Moosavi, S., Xiao, Y., Cheng, W. Y., Bhak, R. H., Tawadrous, M., Capparella, M. R., Montravers, P., \& Duh, M. S. (2018). Retrospective cohort study comparing the risk of severe hepatotoxicity in hospitalized patients treated with echinocandins for invasive candidiasis in the presence of confounding by indication. BMC Infectious Diseases, 18:438.

Wang, R., Qi, X., Yoshida, E.M., Mendez-Sanchez, N., Teschke, R., Sun, M., Liu, X., Su, C., Deng, J., \& Deng, H. (2018). Clinical characteristics and outcomes of traditional Chinese medicine-induced liver injury: A systematic review. Expert Rev. Gastroenterol. Hepatol., $12,425-434$.

Wang, S., Shangguan, Y., Ding, C., Li, P., Ji, Z., Shao, J., Fang, H., Yang, M., Shi. P., Wu, J., Ren, J., Yang, S., Yuan, J., Shi, Y., Li, J., Li, L., \& Xu, K. (2018). Risk factors for acute liver failure among inpatients with anti-tuberculosis drug-induced liver injury. Journal of International Medical Research, 48(1), 1-10. http://doi.org/10.1177/0300060518811512

Whittemore, R., \& Knafl, K. (2005). The integrative review: updated methodology. J Adv Nurs, 52(5), 546-53.

Worland, T., Chin, K. L., Rodrigues, B., \& Nicoll1, A. (2020). A retrospective case-controlled cohort study of inpatient drug induced liver injury: the RIDDLE study. Translational Gastroenterology and Hepatology, 5(33), 1-8. http://dx.doi.org/10.21037/tgh.2019.10.15

World Health Organization. (2020-12-17). ATC/DDD Index 2021. Retirado https://www.whocc.no/atc_ddd_index/ 
Research, Society and Development, v. 10, n. 16, e70101623236, 2021

(CC BY 4.0) | ISSN 2525-3409 | DOI: http://dx.doi.org/10.33448/rsd-v10i16.23236

Woo, H. J., Kimb, H. Y., Choi, E. S., Cho, Y-H., Kima, Y., Lee, J-H., \& Jang, E. (2015). Drug-induced liver injury: A 2-year retrospective study of 1169 hospitalized patients in a single medical center. Phytomedicine, 22(13), 1201-1205. https://doi.org/10.1016/j.phymed.2015.10.002

Xu, H-M., Chen, Y., Xu, J., \& Zhou, Q. (2012). Drug-induced liver injury in hospitalized patients with notably elevated alanine aminotransferase. World J Gastroenterol. 18(41), 5972-5978. http://doi.org/10.3748/wjg.v18.i41.5972

Yang, H.; Guo, D.; Xu, Y.; Zhu, M.; Yao, C.; Chen, C.; \& Jia, W. (2019). Comparison of Different Liver Test Thresholds for Drug-Induced Liver Injury: Updated RUCAM versus Other Methods. Front. Pharmacol. 10, 816. 